\title{
KAMEDIN - Teleconferencing and Automatic Image Analysis for Medical Applications
}

\author{
P. Bernardes, Ch. Busch, M. Groß, J. Miehe, S. Nowacki, A. Will, Ch. Hahn, \\ H. Handels, H. Putzar, E. Rinast and K. Rösler
}

\begin{abstract}
The importance of cooperative work is growing in some medical areas such as radiology. The development of more efficient methods for Computer Supported Cooperative Work (CSCW) is necessary for the introduction of computer support techniques in medical applications. A realisation of CSCW-functions for the support of cooperative work in radiology is being developed in the cooperation project KAMEDIN (Kooperatives Arbeiten und MEdizinische Diagnostik auf Innovativen Netzen). Radiological image data is kept locally in a workstation where it will be exchanged, processed and analysed by two remotely located medical experts using an ISDN connection. Artificial neural networks and high-level image processing procedures are used for classification and 3D reconstruction of different tissues. Kohonen-Feature-Maps are used successfully for the classification task.
\end{abstract}

\section{Introduction}

Nowadays medicine is constantly and progressively developing. Thus new working and cooperation methods are required [3]:

- On the one hand, extended specialisation highly demands consultation and communication, in order to achieve an optimal treatment of the patients. Radiology is an area were this is of high importance;

- On the other hand, the availability of modern image acquisition equipment is usually restricted to university hospitals or comparable medical institutions. 
To achieve these aims CSCW concepts are applied. ISDN is recommendable as a medium of communication because of its relatively low costs and general availability [1]. The ISDN network will soon be available all over Europe due to the standardisation efforts of the European telecommunication companies.

The KAMEDIN project was developed within this context and is financially supported by the Deutsche Telekom. KAMEDIN is a software package designed and developed for UNIX-workstations, which makes it possible to work cooperatively with image data of radiological nature, using the standard ISDN network of the Deutsche Telekom. The image data may be exchanged and subsequently discussed during a cooperative diagnosis session [3]. Another feature is to perform automatic segmentation and image classification of parts of the body from magnetic resonance $(M R)$ and computer tomography $(C T)$ imaging data sets using artificial neural networks [2]. Anatomical objects can be reconstructed in 3D using the segmentation results.

\section{Functionality}

KAMEDIN's functionality is divided into three main aspects:

- local work;

- cooperative work;

- automatic classification and segmentation.

\subsection{Local Work}

The start up for a KAMEDIN session is always a local session.

In order to normalise the handling of image data in KAMEDIN it is necessary to convert the various MR and CT data formats into the KAMEDIN format. The conversion transforms a set of images into a sequence as the basic logical unit. All of the images in the set - stemming from one or multiple data sources - refer to one object, in general a patient. The image data in KAMEDIN format can be analysed in a KAMEDIN session using the radiological basic image processing functions for the sequence representation, observation modus, windowing, density measuring, zooming, etc.

The sequence representation in KAMEDIN can be done by sequence overview or by single image representation. The sequence overview is an image that is created during the conversion of the various MR and CT formats into the KAMEDIN format. The purpose of this image is to achieve a quick overview of the sequence's content. The single image representation is used to analyse each layer of the se- 
quence in more detail. It is also possible to mark the regions of interest (ROIs) that will be used in the automatic classification and segmentation.

Common image representation functions, which are well established in radiology, are included in KAMEDIN. As an example the observation modus allows single and double image viewing. While the single image observation modus is the default, the double image viewing modus must be chosen if it turns out to be necessary to analyse two images of the same or different sequences.

The windowing is necessary for an optimal visual representation of CT and MR images. The representation of $2^{12}$ possible signal values into $2^{8}$ grey values will be calculated once a new window is chosen. As in radiology a window is defined by its center and width. The signal values of the window area will be linearly represented in 256 grey levels. The signal range under the window will be set as the lowest grey value (black) and the signal range over the window will be set as the highest (white). Standard window definitions, i.e. for different data types (MR/CT) and different tissue types (soft tissue and bones), can be stored and reused with other sequences.

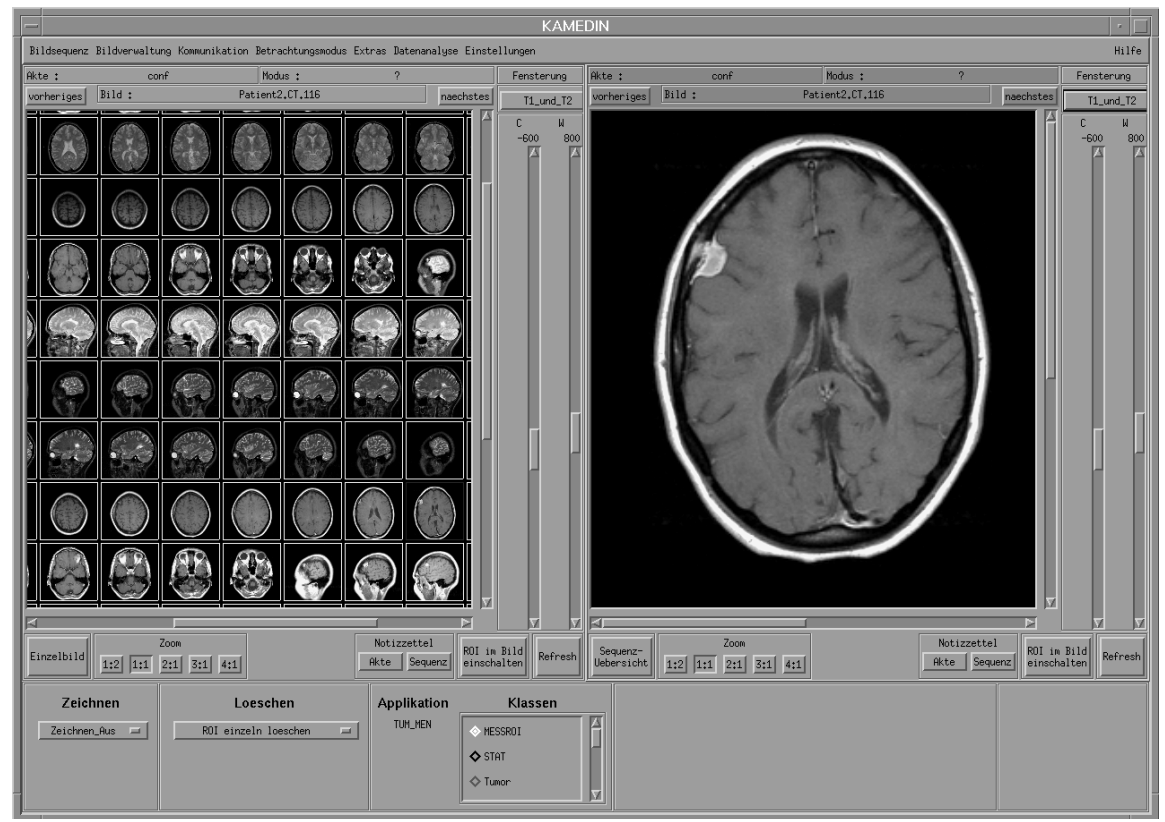

\section{Fig. 1 : A Local Session}

The density measure value of an image point (pixel) and its neighbourhood might be requested. For this purpose the arithmetical average of the affected image points is computed.

A local session can always evolve into a cooperative session where a conference will take place. Conference documents can be combined for a particular remote- 
partner due to function elements such as a file manager and an internal address book. The document contains the data that will be discussed as well as textual annotations for the partner. The document, which is the data base for the cooperative session, must be sent before beginning the conference.

All these fuctionalities of KAMEDIN can be seen in Fig. 1.

\subsection{Cooperative Work}

The cooperative session can be started after having combined and transferred the conference document to a particular remote-partner. During a cooperative session (or teleconference) cooperative work techniques enable two geographically separated specialists to analyse medical image data at the same time [1]. The cooperative work includes multimedial components that enable the simultaneous use and process of textual and audio information. The conference concept can be imagined as a remote-partner taking part in the local session. In this case the remote session as a slave is controlled by the local master session. As a result the local user interface and all local actions are mirrored on the partner's screen.

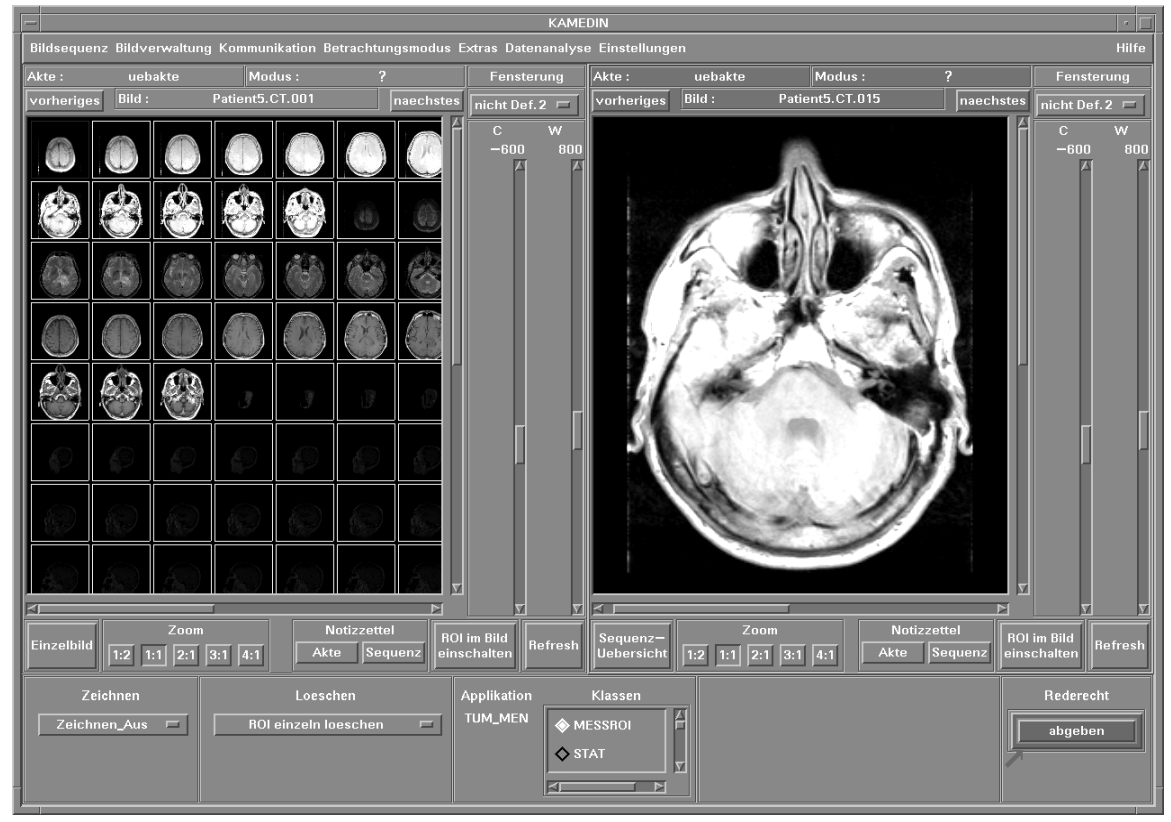

Fig. 2 : A Cooperative Session

At the starting point of a conference the local session of the initiating partner turns into a cooperative session. At the conference end the cooperative session will be transformed into a local session again. For the invited partner the session is 
temporarily limited to the duration of the conference. The partners' actions during the conference will be synchronised by a flow-control mechanism. Fig. 2 is an example of a cooperative session.

During a conference only one partner has the flow-control. With the flow-control it is possible for him to perform all actions interactively. The partner without flowcontrol can only perform two tasks: ask for the flow-control or finish the conference. The exchange of the flow-control must be confirmed. At the cooperative session the conference will be held synchronously. It is not possible to process image data that is not in the conference document, because in this situation it would not be possible to claim for an equal view.

An important aid for the reinforcement of the partner's telepresence is to make a second cursor available. The position of the partner's mouse is always available as a second cursor, so that it is possible to follow remote actions and telepointings.

\subsection{Automatic Classification and Segmentation}

The radiological image data can be analysed automatically using image processing algorithms. The result should support the medical diagnosis. The aim of the classification process is to separate the different tissue types from the original data and to segment the pathological areas [2]. From the medical point of view the main purpose is the segmentation of tumors in image data of the human brain. The result is a classified and colour coded image, where the state and dimension of pathological tissues are evidenced. These results can be visualised three-dimensionally under PHIGS+. A rough description of the anatomical object's surface can be derived from the marching-cubes algorithm [2]. To generate a more precise surface description the Delaunay 3D-triangulation is currently being evaluated [5].

The proceeding steps of the automatic analysis and classification can be divided as follows:

- $\quad$ subdivision in classes;

- $\quad$ establish the training areas or areas of interest;

- feature extraction;

- training;

The subdivision into classes depends on the medical questions and on the represented anatomical objects of the respective acquisition mode. It is possible to subdivide the image data into different tissue types, such as tumor, bone, fat tissue, etc.

In order to train the neural network it is necessary to select a statistically representative image pattern of each class (tissue type). This selection is obtained from the original data by defining areas of interest and should contain a good representation of the defined classes. The radiologist defines the area of interest by pointing out a ROI in the original sequence (see Fig. 3). This work is usually carried out 
during a local session. The expert knowledge is the basis of the quality of an automatic segmentation.

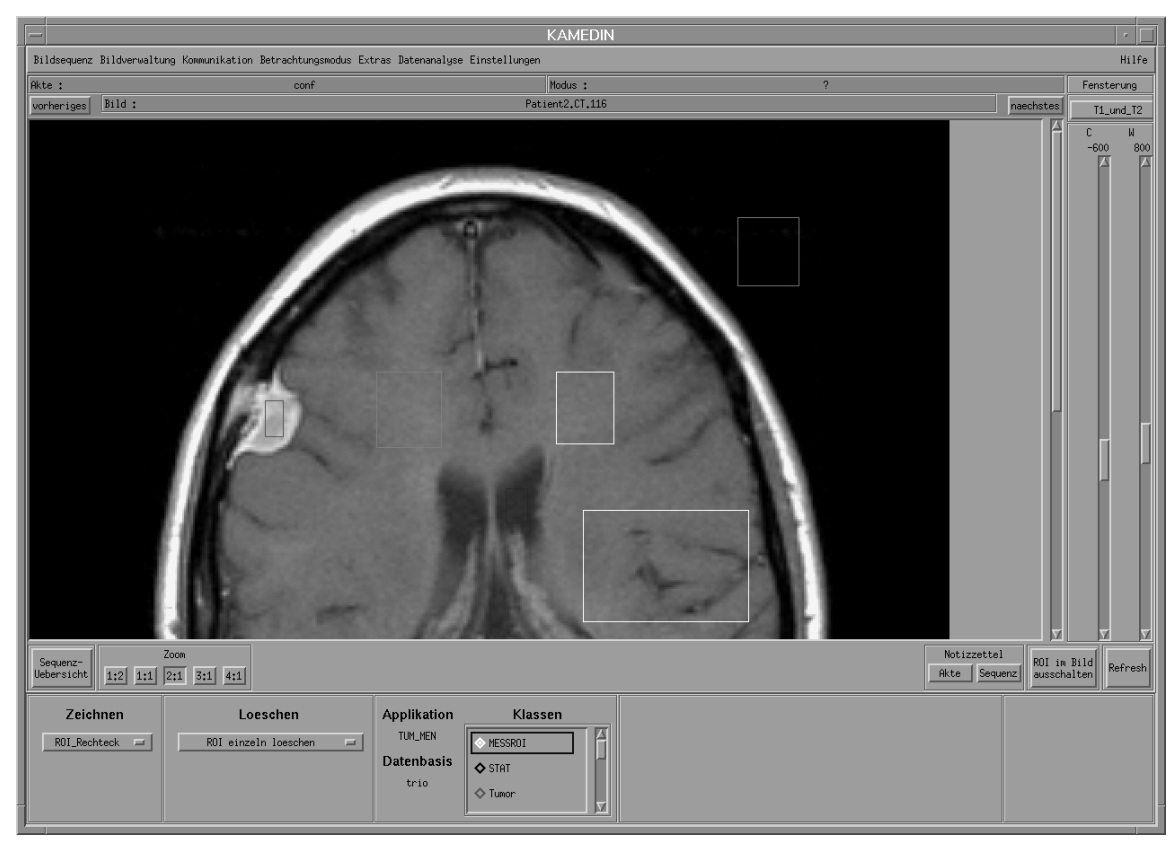

Fig. 3 : Regions of Interest

It is necessary to define ROIs that represent not only tissue types but also the statistically selected samples before beginning the training of the neural network. The representative samples are stored in image databases after having defined all areas of interest.

The local three-dimensional neighbourhood of a volume element must be taken into consideration for the extraction of relevant features. For the analyses of multidimensional volume data, as for example in double or multi-echo sequences, the features of corresponding pixels will be stored in a feature vector.

An artificial neural network will be iteratively adjusted to a classification problem during the training process. Artificial neural networks, such as the MultilayerPerceptron [6] and Kohonen-Feature-Map [4], are available to support the diagnosis with automatic segmentation and classification of different tissue types. The use of a three-dimensional Kohonen-Feature-Map, whose competitive layer is a cube with $6 \times 6 \times 6$ neurons, has proven to be excellent.

\section{Architecture/Implementation}


The important aspects of KAMEDIN's architecture and implementation are:

- the TCP/IP protocol and ISDN;

- the modularity of KAMEDIN;

- the batch-oriented conference preparation;

- the super-computer connection.

\subsection{TCP/IP Protocol and ISDN}

The KAMEDIN-system is a distributed system for teleconferencing purposes that uses the TCP/IP protocol to establish a point-to-point connection. The narrow-band ISDN network with $2 \times 64 \mathrm{kbits} / \mathrm{s}$ from the Deutsche Telekom is used for the data exchange and for the communication during a conference.

Although KAMEDIN was designed for ISDN, it does not depend on ISDN. Due to the use of the network protocol standard TCP/IP, the KAMEDIN-system could be installed on other LAN or WAN networks. Other network types like Ethernet, B-ISDN or the wide-band FDDI would simply impact the rate of data transfer.

\subsection{Modularity}

The KAMEDIN-system was designed as a set of dependent processes. Among these the most important are the daemon, the session manager and the user interface, which coordinate local and cooperative work. Their relationships can be seen in Fig. 4. Their specific functionality will be explained in the next paragraphs.

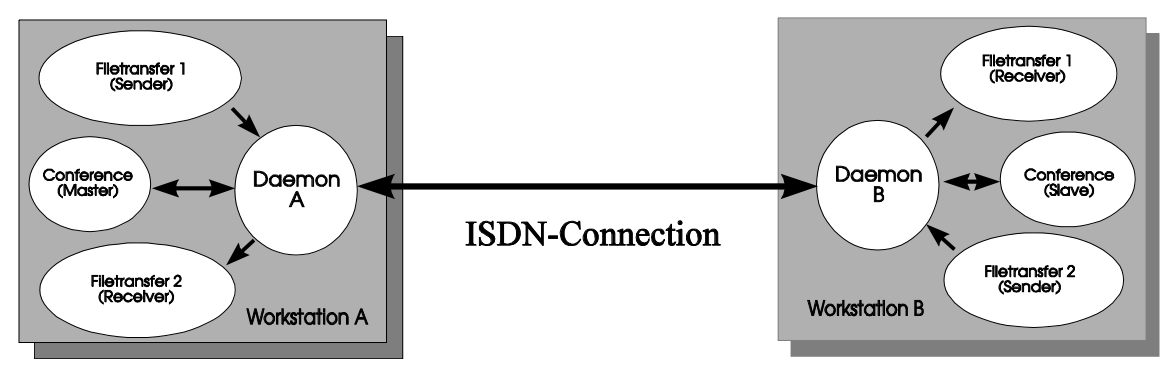

Fig. 4 : The KAMEDIN Architecture

Daemon. The KAMEDIN daemon represents the kernel of the KAMEDIN system architecture. It is the part of the system that guarantees the constant external communication with the system. The daemon's tasks are:

- establish and end the connections to other KAMEDIN systems;

- start the session manager;

- build up a conference; 
- file transfer of the conference documents and batch jobs for the remote supercomputer;

- local distribution of the batch job results;

- manage the transfer capacity of the ISDN connection for the other KAMEDIN modules.

The daemon will be started once and run as a background process. For the communication through other modules it has two communication interfaces. The interfaces enable remote and local modules to send commands to the daemon. These commands will be processed and returned to the local and remote running modules. For the local requests the daemon has a FIFO (first in first out) queue. The communication through remote modules will be achieved via a socket (a socket is a form of inter-process communication in UNIX). A fixed port number or address is attributed to the socket in order to enable communication through other systems.

The daemon receives all commands through this interface. The commands that are interpreted and processed by the daemon are actions like start of a session, building up a connection or initiating a file transfer.

Session Manager. The session manager controls an interactive session of a user in the KAMEDIN system. It executes the notification to the local daemon, allocates the necessary resources, starts the user interface (UI) and controls the actions that will be performed during the KAMEDIN session. The session manager is responsible for the synchronisation of the participating partners during a conference. The resources will be released by the session manager at the end or by stopping the execution under an error condition.

User Interface. The user interface as a dependent process realises all interactions between the user and the system. It includes all functionalities for on-line image processing, such as windowing, zooming, etc. They were described in more detail in subsection 2.1.

Analysis Process. The analysis process realises the image analysis according to the procedures described in subsection 2.3, where the time demanding parts are send to the super-computer.

The KAMEDIN batch-jobs with the necessary data will be sent to the supercomputer and there they will be processed as network queuing system (NQS) jobs. See subsection 3.4.

\subsection{Batch Oriented Conference Preparation}

Since an on-line transfer over the narrow band ISDN with $2 \times 64 \mathrm{kbit} / \mathrm{s}$ of the image data to be analysed (about 130-512 kByte per image, i.e. about 100 MByte per patient with $\mathrm{CT}$ and MR data) would take considerable time during a session, it 
will be sent batch-oriented via ISDN in the conference document before starting a conference. So both conference partners will have the same image data at their disposal. During a session only a limited number of commands must be transferred over the ISDN network. That is an important difference between the KAMEDINsystem and comparable systems.

The processes that participate in the data transfer run with reduced priority in background mode without obstructing the user's work.

\subsection{Super-computer Connection}

The image data analysis using an artificial neural network requires high computing performance. In addition most of the performed operations are vector operations. This is described in detail in [4]. Therefore the KAMEDIN network was linked to a super-computer in order to relieve the local workstation from time consuming processes.

In general a super-computer is capable of processing data fields in an efficient way using vectorization and parallelization of the process. As opposed to the SISD (single instruction single data) computer of the classical von Neumann architecture, the vector processor is called SIMD (single instruction multiple data) computer.

Certain operations in the data fields, as for example addition and multiplication, can be executed in parallel in the elements of the field. The data fields can be also designated as vectors.

The super-computer used in KAMEDIN is a $5400 / 40$ of the firm SiemensNixdorf-Informationssysteme AG (SNI), which is identical to the Fujitsu VP2000. The operating system of the super-computer is the UXP/M. UXP/M is based on the UNIX-system V Release 4, that was extended with components for the vector operations. The NQS is available for the management of time consuming jobs.

The $\$ 400 / 40$ has four scalar processors and two vector processors. In the Siemens terminology they are designated as scalar and vector units, respectively. The scalar unit decodes all instructions, whereby vector operations are transmitted to the vector unit. With this architecture the vector processor can be thought of as a co-processor to the scalar processor (see Fig. 5). In order to optimise the usage of the fast and expensive vector unit, two scalar units have alternating access to each vector unit, where each scalar unit uses its own vector register. The logical separation of scalar and vector units allows parallel processing of scalar and vector instructions. 
Using the architecture of the super-computer a dramatic reduction of the required time for KAMEDIN batch-job99s can be reached. This is made possible with high rates of vectorizable code (transforming for-loops to vector operations) within the analysis process. While the automatic analysis of an MR sequence with 30 slices, $256 \times 256$ pixels each, took up to 463 CPU-minutes for 10,000 cycles on a HP-workstation, the super-computer calculated the same result in $300 \mathrm{CPU}$ seconds. A reduction of the computational time down to $2 \%$ can be reached [7].

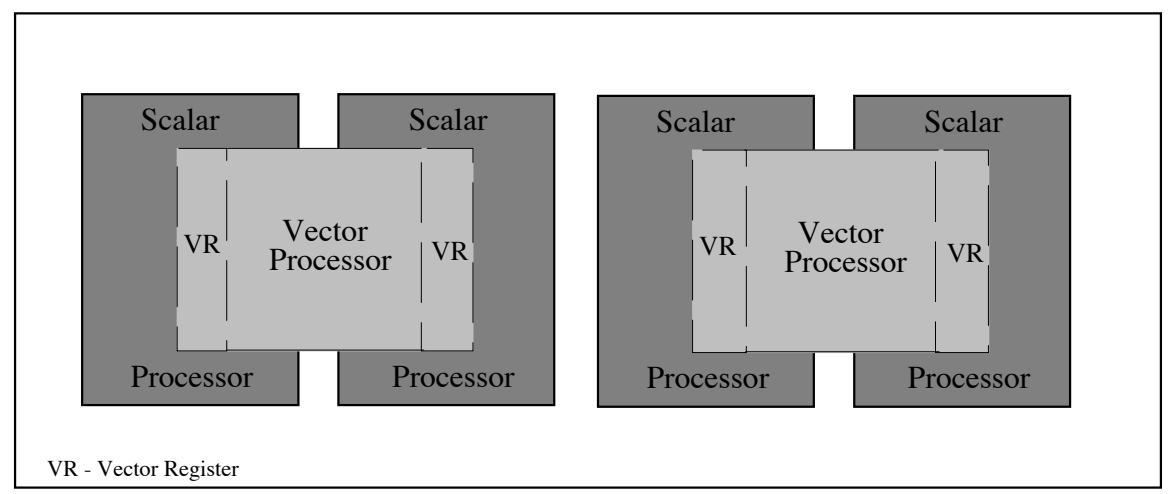

Fig. 5 : The Super-computer Architecture

\section{Conclusions}

The first prototype of KAMEDIN is currently being tested between Darmstadt, Lübeck and Rostock. The prospective users of the KAMEDIN-system will be physicians that need to consult some specialists which work in research departments of hospitals and medical institutions.

\section{References}

1. Ch. Busch, M. Groß, H. Handels, Th. Roß, Ch. Hahn, E. Rinast, K. Rösler, H. Putzar, J. Miehe, S. Nowacki, A. Will, U. von Lucas: Kooperatives Arbeiten und rechnergestützte Ferndiagnostik mittels neuronaler Netze auf ISDN-Leitungen. 38th Annual Meeting of GMDS, 1993. 
2. Ch. Busch, M. H. Groß: Interactive Neural Network Texture Analysis and Visualization for Surface Reconstruction in Medical Imaging, in: Computer Graphics Forum, Vol. 12, no. 3, Conf. Iss., Barcelona, Eds. R.J. Hubbold and R. Juan, 1993.

3. Ch. Hahn, H. Handels, E. Rinast, Ch. Busch, M. Groß, J. Miehe, S. Nowacki, A. Will, K. Rösler, H. Putzar: Telekonferenzen über ISDN mit dem Softwaresystem KAMEDIN, presented in the Workshop of Digital Image Processing in Medicine, University of Freiburg, 2nd-4th March 1994.

4. T. Kohonen: The Self-Organizing Map. Proceedings of the IEEE, Vol. 78, No. 9, pp. 1464-1480, 1990.

5. H. Müller, A. Klingert: Surface Interpolation from Cross Sections, in: Focus of Scientific Visualization, H. Hagen, H. Müller and G. Nielson (eds.), Springer, 1993, pp. 139-185.

6. D. E. Rumelhart, J. L. McClelland: Parallel Distribution Processing. Explorations in the Microstructure of Cognition, Vol. 1 and 2., MIT Press, Cambridge, Massachusetts, 1986.

7. D. Willet: Grundlagen, Konzeption und Realisierung multidimensionaler Bildanalyse mit Neuronalen Netzen unter Verwendung eines Vectorrechners. Diploma Theses, TH Darmstadt, 1994. 